\title{
Avaliação dos Registros de Enfermagem Acerca das Manifestações Clínicas de Cardiotoxicidade
}

\author{
Evaluation of Nursing Records on Clinical Manifestations of Cardiotoxicity
}

Mayara Davila Borges ${ }^{1}$ * Rafaela de Oliveira da Silva ${ }^{2}$ Karla Biancha Silva de Andrade 3 * Sandra Regina Maciqueira Pereira ${ }^{*}$ Luana Ferreira de Almeida ${ }^{5}$ Danielle de Mendonça Henrique 6

\begin{abstract}
RESUMO
Esta pesquisa objetivou avaliar os registros de enfermagem em um ambulatório de quimioterapia, acerca das manifestações clínicas de cardiotoxicidade e apresentar uma proposta de roteiro de informações direcionada aos registros dos cuidados nessa clientela. Trata-se de uma pesquisa documental, retrospectiva por análise do prontuário e avaliação quantitativa. Os dados foram coletados em formulário próprio contendo 4 variáveis direcionadas às características da população e 15 variáveis relacionadas às possíveis manifestações clínicas de cardiotoxicidade, obedecendo as normas éticas e analisados por meio da estatística descritiva. A proposta de um roteiro de informações foi descrita a partir de um quadro contendo variáveis sociodemográficas e de elementos específicos do tratamento, elaboradas a partir da revisão de literatura desta pesquisa. Apesar da potencial toxicidade do tratamento quimioterápico, não houve registro do medicamento quimioterápico utilizado em $12(50 \%)$ prontuários avaliados e não foi encontrado nenhum registro de enfermagem sobre possíveis sinais de cardiotoxicidade. Conclui-se que a quimioterapia pode desenvolver reações tóxicas, como por exemplo, a cardiotoxicidade. O conhecimento sobre os possíveis sinais e sintomas de toxidade cardíaca, bem como dos fatores de risco e adequado registro dessas informações pela enfermagem, tornam-se importantes para o alcance de uma documentação clínica com qualidade.
\end{abstract}

\section{Palavras-chaves: Cardiotoxicidade; Quimioterápicos; Registro de Enfermagem}

\begin{abstract}
This research aimed to evaluate the nursing records that work in the chemotherapy outpatient clinic about the clinical manifestations of cardiotoxicity and to present a proposal of information route directed to the records of care in this clientele. It is a documentary research, retrospective by analysis of the medical record and quantitative evaluation. The data were collected in a form containing 4 variables directed to the characteristics of the population and 15 variables related to the possible clinical manifestations of cardiotoxicity, obeying ethical norms and analyzed through descriptive statistics. The proposal of an information roadmap was described from a table containing sociodemographic variables and specific elements of the treatment, elaborated from the literature review of this research. Despite the potential toxicity of the chemotherapy treatment, there was no record of the drug used in 12 (50\%) medical records and no nursing record was found on possible signs of cardiotoxicity. It is concluded that chemotherapy can develop toxic reactions, such as cardiotoxicity. Knowledge about the possible signs and symptoms of cardiac toxicity, as well as the risk factors and adequate recording of this information by nursing, become important for the reach of clinical documentation with quality, that when this looks for the service. It is necessary to create concrete actions that meet the specific needs of this group, as well as prepare the team to deal with this population providing linkage and integral attention to adolescent health.
\end{abstract}

Keywords: Cardiotoxicity; Chemotherapeutics; Nursing Record.

${ }^{1}$ Enfermeira. Mestranda do Programa Acadêmico em Ciências do Cuidado em Saúde (PACCS) da Universidade Federal Fluminense-UFF. Especialista em Terapia Intensiva pelo programa de Residência Multiprofissional em Saúde - UFF. Graduada em Enfermagem pela Universidade do Estado do Rio de Janeiro - UERJ: mayaraborgesrb@hotmail.com

${ }^{2}$ Especialista na modalidade de Residência em Enfermagem em Saúde da Família pela Universidade do ${ }^{3}$ Estado do Rio de Janeiro - UERJ. Graduada em Enfermagem-UERJ: rafaelaoli03@gmail.com

${ }^{3}$ Enfermeira. Doutora. Professora Adjunta da Faculdade de Enfermagem da Universidade do Estado do Rio de Janeiro Chefe do Departamento de Enfermagem Médico-cirúrgica: karla.biancha@gmail.com

${ }^{4}$ Enfermeira. Doutora. Professora Adjunta da Faculdade de Enfermagem da Universidade do Estado do Rio de Janeiro. Subchefe do Departamento de Enfermagem Médico-cirúrgica: sandregina@gmail.com

${ }^{5}$ Doutora. Professora Adjunta da Faculdade de Enfermagem da Universidade do Estado do Rio de Janeiro:Iuana.almeida3011@gmail.com.

${ }^{6}$ Enfermeira. Doutora. Professora Adjunta do Departamento de Enfermagem Médico Cirúrgico da Faculdade de Enfermagem da Universidade do Estado do Rio de Janeiro: danimendh@gmail.com 


\section{INTRODUÇÃO}

Doenças e agravos não transmissíveis são as principais causas de adoecimento e mortes na população mundial. Estima-se que em 2008, ocorreram 36 milhões de mortes decorrentes dessas doenças, com destaque para as doenças cardiovasculares e câncer, e tendo maior impacto nos países pouco desenvolvidos. A transição demográfica e epidemiológica das últimas décadas, apontam o câncer com maior carga nas próximas décadas $^{(1)}$.

A estimativa para o Brasil no biênio 20182019 aponta a ocorrência de 600 mil casos novos de câncer. Com exceção para o câncer de pele não melanoma, previsto para 170 mil casos novos, os outros tipos de cânceres serão cerca de 420 mil, os quais os mais incidentes serão os tumores de próstata (68 mil), mama feminina (60 mil), cólon e reto (35 mil), pulmão (30 mil), estômago (21 mil) e colo do útero $(16 \mathrm{mil})^{(1)}$.

Um dos mais importantes e promissores tratamentos contra a doença oncológica é a terapêutica com quimioterapia antineoplásica, a qual é baseada na utilização de agentes químicos, seja isolado ou em combinação. É uma modalidade de tratamento sistêmico, diferente da cirurgia e radioterapia, as quais são mais antigas e de atuação localizada. A sua capacidade de atuação sistêmica possibilitou a cura de leucemias e linfomas, além de permitir o tratamento de metástases (definida como progressão da doença em outro órgão, através da migração por via sanguínea ou linfática das células cancerosas) não detectáveis ${ }^{(2-3)}$.

A quimioterapia pode ser empregada com a finalidade curativa, quando o tratamento sistêmico é o definitivo, como, por exemplo, para os linfomas e leucemias; pode ser uma terapia adjuvante, quando o tratamento objetiva aumentar a chance de cura após determinado procedimento cirúrgico, como exemplo tratamento de câncer de mama e pulmão; pode atuar como neoadjuvante, quando ela é empregada antes do tratamento curativo, visando menor radicalidade no procedimento cirúrgico ou reduzir o risco de metástases e, paliativa, quando o objetivo é a paliação da doença ${ }^{(2-3)}$.

A terapêutica da doença oncológica com quimioterapia pode ser realizada em hospitais especializados, unidades ambulatoriais e clínicas oncológicas $^{(3-4)}$.

Nas últimas décadas os tratamentos antineoplásicos têm evoluído drasticamente e melhoraram substancialmente o prognóstico dos pacientes oncológicos. Porém, o uso contínuo dos agentes quimioterápicos na prática clínica pode gerar controvérsia, devido aos seus potenciais efeitos adversos ao nível cardiovascular nos pacientes tratados que sobrevivem à doença oncológica(4). $^{(4)}$.

As principais complicações decorrentes do tratamento antineoplásico são: toxicidade hematológica, toxicidade gastrointestinal, cardiotoxicidade, hepatotoxicidade, toxicidade pulmonar, neurotoxicidade, disfunção reprodutiva, toxicidade vesical e renal, alterações metabólicas, toxicidade dermatológica, reações alérgicas e anafilaxia e fadiga ${ }^{(3)}$.

Dessa forma as doenças cardiovasculares nos pacientes com câncer são eventos cada vez mais frequentes. Nos últimos anos, os progressos no tratamento oncológico originaram, também, uma maior exposição dos pacientes aos fatores de riscos cardiovasculares, além de muitos agentes quimioterápicos apresentarem capacidade cardiotóxica $^{(5)}$.

Os efeitos cardiotóxicos dos quimioterápicos estão relacionados ao tempo de exposição e à concentração plasmática da droga. Além de fatores de risco como, extremos de idade, disfunção ventricular prévia, hipertensão arterial, diabetes, uso de quimioterápicos associados, radioterapia mediastinal e suscetibilidade genética, contribuem para o desenvolvimento da cardiotoxicidade ${ }^{(5-6)}$.

A definição de cardiotoxicidade é baseada nas medidas de fração de ejeção do ventrículo esquerdo (FEVE), a qual em indivíduos normais, estas medidas são entre $55 \%$ ou mais. A cardiotoxicidade é classificada em: a) grau I, quando ocorre redução assintomática da FEVE entre $10 \%$ a $20 \%$; b) grau II quando ocorre redução da FEVE abaixo de $20 \%$ e; c) grau III quando há 


\section{ARTIGO ORIGINAL}

insuficiência cardíaca sintomática (dispneia, cansaço, edema) ${ }^{(6-7)}$.

A apresentação clínica da cardiotoxicidade pode ser aguda ou subaguda, quando ela se desenvolve no início ou em até 14 dias do término do tratamento e crônica, a qual é subdividida em dois subtipos: a) quando os sintomas clínicos aparecem dentro de um ano após o término do tratamento e b) quando as manifestações clínicas surgem após um ano do término do tratamento da quimioterapia $^{(7)}$.

Desta forma, o tratamento com quimioterapia requer da equipe de saúde a interpretação de seu benefício imediato e, também, a identificação de fatores de risco, como radioterapia de mediastino, cardiopatia prévia, tratamento oncológico anterior, assim como a identificação precoce e controle dos sinais de toxidade.

E essas ações precisam estar registradas pela equipe de saúde a fim de monitorar e avaliar a assistência prestada, bem como dar continuidade ao processo do cuidado.

No Brasil, desde 2002 é exigido que todo profissional de enfermagem documente todas as etapas do processo assistencial em que o cuidado de enfermagem ocorra. Hoje, após quinze anos, alguns hospitais brasileiros ainda lutam para implementar essa regulação ${ }^{(8)}$.

A boa documentação clínica permeia a segurança do paciente, e é a segunda meta do Programa Nacional de Segurança do Paciente, instituído pela Portaria no 529, de 1 de Abril de 2013, a qual visa a comunicação efetiva entre os profissionais de saúde ${ }^{(9)}$. Ela permite o registro da assistência, oferecendo dados para os diagnósticos de enfermagem, os quais direcionam as metas que deverão ser alcançadas e as intervenções que precisarão ser traçadas, permitindo a avaliação e continuidade do cuidado ${ }^{(10-11)}$.

Os registros de enfermagem consistem em uma forma de comunicação escrita que contém informações pertinentes sobre o paciente e os cuidados fornecidos pela equipe de enfermagem. São elementos essenciais na documentação da assistência, considerando que, quando redigidos adequadamente, possibilitam a comunicação efetiva entre a equipe de enfermagem e os demais profissionais envolvidos no cuidar ${ }^{(11-12)}$.

Além disso, servem de base para a elaboração do plano assistencial ao paciente, fornecem subsídios para a avaliação da assistência prestada, fazem parte da evolução do paciente favorecendo e colaborando com a auditoria de enfermagem, $o$ ensino e pesquisa em enfermagem $^{(12)}$.

A ausência de registros pode implicar, na duplicação de procedimentos executados, dificuldade de acompanhamento dos cuidados prestados, e até na não execução de determinada atividade, podendo colocar em risco a própria recuperação do paciente ${ }^{(7-10-11-13)}$.

Desta forma, para promover a continuidade do planejamento dos cuidados de enfermagem em suas diferentes fases, o registro deve constar de impressos devidamente identificados com os dados do paciente, ser claro e objetivo, com identificação do autor, feito de forma legível, sem rasuras, fazer parte do prontuário e favorecer elementos administrativos e clínicos para a auditoria de enfermagem ${ }^{(14)}$.

Sendo assim, o registro de enfermagem é um aspecto primordial da prática de enfermagem, o qual precisa estar em constante evolução, a fim de aprimorar sua estrutura e qualidade, e deve ter foco no impacto favorável do cuidado e na segurança do paciente $\mathrm{e}^{(11-14)}$.

Registros de enfermagem completos delimitam as ações de cuidar desenvolvidas no interior de um processo de trabalho e indicam o modo como os enfermeiros e suas equipes prestam, representam e significam o cuidado de enfermagem que contém indícios reveladores da história e da cultura profissional ${ }^{(8)}$.

Nesse sentido, esta pesquisa teve como problema do estudo: Como são os registros de enfermagem no ambulatório de quimioterapia de um hospital Universitário no estado do Rio de Janeiro?

Pressupõe-se que a equipe de enfermagem que atua no ambulatório de quimioterapia do cenário em questão não realiza adequadamente os registros nos prontuários acerca das manifestações clínicas de cardiotoxicidade, dentre outras 
possíveis alterações que o paciente está sujeito, tendo em vista que a mesma não possui um roteiro de informações para seus registros.

E para responder ao problema de pesquisa, este estudo teve como objetivos avaliar os registros de enfermagem em um ambulatório de quimioterapia acerca das manifestações clínicas de cardiotoxicidade e apresentar uma proposta de roteiro de informações direcionado aos registros dos cuidados nessa clientela.

\section{MÉTODO}

Trata-se de uma pesquisa documental, retrospectiva, que utilizou a técnica de coleta de dados por análise do prontuário do paciente e avaliação quantitativa dos mesmos.

O campo da pesquisa foi o ambulatório de quimioterapia de um Hospital Universitário, situado no município do Rio de Janeiro, que funciona em dias úteis no período da manhã e tarde. A equipe de enfermagem do ambulatório é composta por três técnicos e quatro enfermeiros. São atendidos em média cinquenta pacientes por dia. O setor possui uma farmácia que funciona apenas na parte da manhã preparando as medicações de acordo com a prescrição de cada paciente. A coleta de dados ocorreu no período de outubro a novembro de 2015.

Foram avaliados 381 prontuários e selecionados 24 prontuários que obedeceram aos seguintes critérios de inclusão: prontuários dos pacientes submetidos à quimioterapia de janeiro de 2014 a setembro de 2015; idade maior que dezoito anos e os registros contendo folha de evolução padrão da instituição. Foram excluídos os prontuários de pacientes com doença arterial coronariana, fibrilação atrial, arritmas ventriculares e tromboembolismo previamente ao tratamento, prontuários incompletos e indisponíveis no período da coleta dos dados.

Para a coleta dos dados nos prontuários, foi utilizado um formulário próprio, contendo as quatro variáveis direcionadas às características da população: idade, sexo, diagnóstico, quimioterápicos usados no tratamento oncológico e tempo de tratamento e 15 variáveis relacionadas às possíveis manifestações clínicas de cardiotoxicidade: insuficiência cardíaca, hipertensão arterial sistêmica, doença pericárdica, arritmias ventriculares e supraventriculares, isquemia miocárdica aguda com ou sem supra de segmento ST, disfunção ventricular esquerda assintomática, eventos tromboembólicos, tosse não produtiva, dispneia e/ou estertores pulmonares, edema de extremidades, cianose, diminuição da amplitude do pulso periférico, taquicardia, confusão, agitação e torpor.

Para apresentar a proposta de um roteiro de informações direcionadas aos registros de enfermagem aos pacientes em tratamento quimioterápico ambulatorial, foram selecionadas variáveis referentes às informações relacionadas às características sociodemográficas, bem como elementos específicos do tratamento, tais como quimioterápico em uso, números de ciclos realizados, dose da medicação, medicações concomitantes, possíveis reações clínicas do paciente relacionadas ao tratamento quimioterápico, exames complementares prévios, a fim de proporcionar melhor facilidade para o registro da assistência prestada nessa clientela.

A pesquisa obedeceu aos critérios éticos da resolução n. $0466 / 2012^{(15)}$ do Conselho Nacional de Saúde que visa assegurar os direitos e deveres que dizem respeito aos participantes da pesquisa, à comunidade científica e ao Estado. Este estudo tem aprovação pelo Comitê de Ética e Pesquisa CEP/HUPE, parecer $n .0$ 1.310.830.

Os dados da pesquisa foram tabulados e analisados por meio da estatística descritiva.

\section{RESULTADOS}

Foram analisados 381 prontuários de pacientes do ambulatório de quimioterapia de um Hospital Universitário do Rio de Janeiro. Destes, apenas 24 prontuários cumpriram os critérios de inclusão. Os demais 357 prontuários foram excluídos devido às condições de: prontuários sem folha de evolução do setor de quimioterapia (137), prontuários sem a folha de evolução padrão (53), doenças crônicas antes do início do tratamento quimioterápico (134) e prontuários anteriores a 2014 (33). 


\section{ARTIGO ORIGINAL}

Dos 24 prontuários selecionados foram identificados 12 (50\%) do sexo feminino e 12 (50\%) do sexo masculino e $92 \%$ (22) dos pacientes tinham entre 41 a 80 anos. Nesta pesquisa foi identificado um percentual baixo $(4 \%-1)$ para a faixa etária entre 21 e 30 anos e nenhum percentual para a faixa etária entre 31 e 40 anos.

Neste estudo foi possível observar que a maior porcentagem de usuários que realizam tratamento quimioterápico ambulatorial foi entre a faixa etária de 40 a 80 anos, evidenciando a relação do aumento da expectativa de vida da população com as doenças crônico-degenerativas, corroborando com informações anteriores.

Esses achados vão ao encontro de que, o processo global de industrialização conduziu a redefinição de padrões de vida com uniformização das condições de trabalho, nutrição e consumo. Paralelamente, deu-se uma significativa alteração na demografia mundial, devido à redução nas taxas de mortalidade e natalidade e, consequentemente, aumento da expectativa de vida e envelhecimento populacional $^{(16)}$.

Esse processo de mudança demográfica, denominado de envelhecimento populacional, associado às transformações nas relações entre as pessoas e seu ambiente, trouxe uma importante alteração no perfil de morbimortalidade, colocando as doenças crônico-degenerativas, como a doença oncológica, como novo centro de atenção dos problemas de saúde da população brasileira, em detrimento da redução da ocorrência das doenças infectocontagiosas ${ }^{(16)}$.

Outro dado relevante a ser tratado acerca dos prontuários analisados foi a alta taxa de hipertensão arterial sistêmica (HAS), encontrada em $100 \%$ dos prontuários selecionados neste estudo. A HAS tem alta prevalência e baixas taxas de controle, sendo considerada um dos principais fatores de risco modificáveis e um dos mais importantes problemas de saúde pública. A mortalidade por doença cardiovascular aumenta progressivamente com a elevação da pressão arterial a partir de 115/75 mmHg de forma linear, contínua e independente ${ }^{(17)}$.

Entende-se assim que, pacientes portadores de HAS quando são submetidos à quimioterapia com potencial risco cardiotóxicos possuem maior probabilidade de desenvolver a cadiotoxicidade ou apresentar manifestações clínicas da mesma ${ }^{(4-6)}$.

A Tabela a seguir expõe os diagnósticos de câncer dos prontuários selecionados de acordo com o sexo.

Tabela 1. Diagnósticos de câncer encontrados nos prontuários selecionados de pacientes em tratamento quimioterápicos de um Hospital Universitário, RJ, Brasil, 2015.

Fonte: dados da pesquisa.

\begin{tabular}{llll}
\hline Doenças crônicas & Feminino & Masculino & Total \\
\hline Câncer de ovário & 2 & - & 2 \\
Câncer de pâncreas & 2 & 0 & 2 \\
Câncer de colón & 1 & 1 & 2 \\
Câncer de pulmão & 1 & 2 & 3 \\
Sarcoma de Kapossi & 0 & 1 & 1 \\
Câncer de mama & 3 & 0 & 3 \\
Câncer de estômago & 1 & 0 & 1 \\
Adenocarcinoma de & 1 & - & 1 \\
endométrio & & & \\
Câncer de timo & 0 & 1 & 1 \\
Câncer de próstata & - & 2 & 2 \\
Seminoma clássico & - & 1 & 1 \\
Tumor de testículo & - & 1 & 1 \\
Câncer de canal anal & 1 & 0 & 1 \\
Lipossarcoma & 0 & 1 & 1 \\
mixóide & & 1 & 24 \\
Câncer de bexiga & 0 & 1 & \\
Carcinoma escamo- & 0 & & \\
colunar de laringe & & 12 & \\
Total & 12 & & \\
\hline & & & 1 \\
\hline
\end{tabular}

Esses achados corroboram com a incidência sobre os tipos de câncer no Brasil. De acordo com os dados do Instituto Nacional do Câncer (INCA), excluindo os tumores de pele não melanoma, o câncer de próstata é o mais incidente entre os homens em todas as regiões do país, com 91,24/ 100 mil no Sul, 88,06/ 100 mil no Sudeste, 62,55/ 100 mil no Centro-Oeste, 47,46/ 100 mil no Nordeste e 30,16/ 100 mil no Norte. Já nas mulheres, o câncer de mama é o mais frequente sendo nas regiões Sudeste $(71,18 / 100 \mathrm{mil})$, Sul (70,98/ $100 \mathrm{mil})$, Centro-Oeste (51,30/ $100 \mathrm{mil})$ e Nordeste (36,74/ $100 \mathrm{mil})$. Na região Norte, é o segundo tumor mais incidente (21,29/ 100 mil) $17^{(1)}$. 
Além disso, o câncer de pulmão em homens é o segundo mais frequente nas regiões Sul (33,62/ 100 mil) e Centro-Oeste (14,03/ 100 mil). Nas regiões Sudeste $(18,51 / 100 \mathrm{mil})$, Nordeste $(9,01 / 100 \mathrm{mil})$ e Norte (7,69/ $100 \mathrm{mil}), \mathrm{o}$ terceiro ${ }^{(1-17)}$.

Assim, pela análise dos prontuários selecionados, o câncer de mama e o de próstata são os mais encontrados nas mulheres e nos homens respectivamente.

O Gráfico 1 trata o quimioterápico utilizado para o tratamento do câncer no cenário estudado:

Gráfico 1. Quimioterápicos registrados nos prontuários de pacientes em tratamento ambulatorial em um Hospital Universitário, RJ, Brasil, 2015.

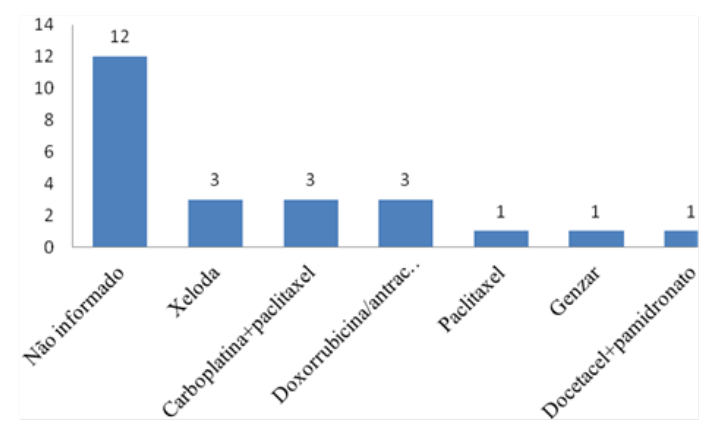

Fonte: dados da pesquisa.

Observou-se que a antraciclina (doxorrubicina) e o paclitaxel, em uso concomitante com a carboplatina, foram encontradas em três prontuários para tratamentos antineoplásicos. Já o docetaxel, em uso concomitante com o pamidronato, foi encontrado para tratamento em apenas um prontuário, assim como o paclitaxel em uso isolado.

Os quimioterápicos mais utilizados no tratamento do câncer e que possuem um percentual de cardiotoxicidade considerável são as antraciclinas (doxorrubicina, epirrubicina, idarrubicina) $5 \%$ a $35 \%$ dos casos, agentes alquilantes, (ciclofosfamida, ifosfamida) $5 \%$ a $25 \%$ dos casos e agente antimicrotúbulos (docetaxel, paclitaxel) $2 \%$ a $10 \%$ dos $\operatorname{casos}^{(7)}$. Ademais, os fármacos antineoplásicos, como as antraciclinas podem causar predominantemente lesões irreversíveis ao miocárdio(4-6).

Além disso, a duração da administração também poderá provocar risco relativo para desenvolvimento de cardiotoxicidade durante ou após a terapia oncológica ${ }^{(4-18)}$.

Sendo assim, vê-se a necessidade de acompanhamento cardiológico dos pacientes que estão em uso de antraciclina (doxorrubicina), docetaxel e paclitaxel com o intuito de identificar qualquer tipo de alteração cardiológica que pudesse comprometer a vida do paciente ${ }^{(3-6-19)}$.

No entanto, mesmo existindo a correlação do uso de quimioterápicos com potencial risco cardiotóxico, não foi encontrado registro sobre o nome da droga ou protocolo utilizado em 12 prontuários. Também não foi encontrado nenhum registro sobre possíveis manifestações clínicas de cardiotoxicidade, assim como os sinais e sintomas de outras toxicidades.

Visto que a administração de quimioterápicos está relacionada diretamente ao potencial risco cardiotóxico é de suma importância que a enfermagem, no cuidado, avalie o paciente antes, durante e após administração desses medicamentos, com o intuito de avaliar qualquer tipo de alteração clínica indicativa de cardiotoxicidade, ou até mesmo seus sintomas préclínicos $^{(3-4-6-19)}$.

Os cuidados de enfermagem essenciais para avaliação do paciente oncológico, com foco na identificação de possíveis alterações clínicas, inclusive de cardiotoxicidade, a verificação dos sinais vitais, peso e altura; avaliação dos resultados dos exames realizados; notificação de intercorrências ao médico; intervenção imediata na ocorrência de efeitos colaterais que possam surgir durante a administração das drogas; e realizar o registro de enfermagem integral do processo de cuidado, bem como as intercorrências, fazendo este, parte do prontuário do paciente $\mathrm{e}^{(3-6-9-19-20)}$.

Entende-se que os registros são componentes imprescindíveis no processo assistencial visto que, quando documentados com qualidade, retratam a realidade na qual o cuidado foi oferecido. Assim, possibilitam a comunicação 


\section{ARTIGO ORIGINAL}

efetiva entre a equipe de saúde, bem como favorecem a realização de pesquisas, auditorias, processos jurídicos e planejamento ${ }^{(20)}$.

Além disso, metade das informações inerentes ao cuidado do paciente são fornecidas pela equipe de enfermagem e é indiscutível a necessidade de registros adequados e frequentes no prontuário do cliente ${ }^{\text {(08-11-13-14). }}$

Os registros de enfermagem, quando escassos e inadequados, comprometem a assistência prestada ao paciente, assim como à instituição e à equipe de enfermagem. E ainda compromete a segurança do paciente, impactando na mensuração dos resultados assistenciais sobrevindos da prática da enfermagem ${ }^{(11-13-20)}$.

Um estudo com análise de prontuários mostrou que durante coleta e análise dos dados, foi possível observar que os registros da equipe de saúde são falhos ${ }^{(21)}$.

Grande parte das informações registradas pela enfermagem limita-se ao registro de dados apresentados pelo paciente, demonstrando a necessidade da sistematização dos registros de enfermagem, para que este seja realizado adequadamente, proporcionando uma assistência mais completa ${ }^{(8-13-19-20)}$.

Do mesmo modo que os registros nos prontuários incompletos em relação aos cuidados ao paciente, em sua maioria, não satisfazem os requisitos definidos pelas instituições de saúde ou conselhos profissionais. As anotações de enfermagem registradas devem ser arquivadas nos prontuários fornecendo informações referentes ao usuário e a assistência prestada pelo profissional ${ }^{(11-}$ 12-22).

Desta forma os registros em prontuário representam um indicador da qualidade dos cuidados fornecidos, além de se constituir em instrumento para respaldo ético e legal aos profissionais responsáveis pelo cuidado, assim como na segurança do paciente, evitando complicações indesejáveis e evitáveis ${ }^{(8-17-19-22)}$.

Reconhecendo a importância da qualidade dos registros de enfermagem sobre os cuidados prestados aos pacientes submetidos à quimioterapia, o quadro 1 apresenta uma proposta de roteiro para informações necessárias sobre a assistência prestada nessa clientela, a partir das variáveis apresentadas e discutidas nesse artigo.

Quadro 1. Proposta de roteiro com variáveis necessárias aos registros das informações sobre a assistência prestada aos pacientes submetidos a quimioterapia ambulatorial em um Hospital Universitário, RJ, Brasil, 2015.

\begin{tabular}{|c|c|}
\hline $\begin{array}{l}\text { Variáveis para } \\
\text { informações sobre: }\end{array}$ & Justificativa \\
\hline $\begin{array}{l}\text { 1. Identificação do } \\
\text { paciente }\end{array}$ & $\begin{array}{l}\text { Erros podem ocorrer no } \\
\text { momento de admissão do } \\
\text { paciente no sistema de } \\
\text { cuidado, através de falhas } \\
\text { nos dados do cadastro do } \\
\text { paciente, podendo } \\
\text { comprometer toda a } \\
\text { segurança no processo } \\
\text { assistencial }{ }^{(20,23)} \text {. Em } \\
\text { prática, a identificação do } \\
\text { paciente é uma etapa do } \\
\text { cuidado de enfermagem } \\
\text { que não recebe a devida } \\
\text { atenção, podendo } \\
\text { interferir nas demais } \\
\text { etapas, essenciais para } \\
\text { garantia da qualidade e } \\
\text { segurança do serviço } \\
\text { prestado(23). Essas falhas } \\
\text { podem resultar desfechos } \\
\text { ruins para o indivíduo, tais } \\
\text { como incapacidades, } \\
\text { internações prosa } \\
\text { desnecessárias, expô-lo a } \\
\text { mais procedimentos e } \\
\text { medidas terapêuticas e, } \\
\text { até mesmo provocar a } \\
\text { morte }{ }^{(20-23-24-25) .}\end{array}$ \\
\hline $\begin{array}{l}\text { 2. Protocolo } \\
\text { quimioterápico/Dosage } \\
\mathrm{m}\end{array}$ & $\begin{array}{l}\text { Os quimioterápicos atuam } \\
\text { afetando os processos } \\
\text { básicos da divisão celular, } \\
\text { assim, muitos sintomas } \\
\text { colaterais desta terapia } \\
\text { medicamentosa estão } \\
\text { diretamente relacionados } \\
\text { ao próprio mecanismo de } \\
\text { atuação. Desta forma, } \\
\text { saber o protocolo e sua }\end{array}$ \\
\hline
\end{tabular}




\begin{tabular}{|c|c|}
\hline & $\begin{array}{l}\text { dosagem, auxilia o } \\
\text { enfermeiro prever a } \\
\text { intensidade dos sintomas } \\
\text { tóxicos gastrointestinais, } \\
\text { bem como a probabilidade } \\
\text { do desenvolvimento de } \\
\text { cardiotocixidade }{ }^{(3-4-6-18-10)} \text {. }\end{array}$ \\
\hline 3. Sinais Vitais & $\begin{array}{l}\text { São indicadores da } \\
\text { capacidade do organismo } \\
\text { em regular a temperatura, } \\
\text { oxigenar os tecidos e } \\
\text { manter fluxo sanguíneo, } \\
\text { bem como desconfortos } \\
\text { relacionados à dor. A } \\
\text { verificação dos sinais vitais } \\
\text { antes, durante e após a } \\
\text { administração dos } \\
\text { quimioterápicos para a } \\
\text { essencial de } \\
\text { identificação precoce de } \\
\text { alterações súbitas na } \\
\text { condição clínica do } \\
\text { paciente }{ }^{(6-7-24)} \text {. }\end{array}$ \\
\hline $\begin{array}{l}4 . \\
\text { Comorbidades/Fatores } \\
\text { de risco }\end{array}$ & $\begin{array}{l}\text { Para o diagnóstico da } \\
\text { miocardiopatia associada } \\
\text { à quimioterapia, é } \\
\text { importante definir a classe } \\
\text { e o quimioterápico } \\
\text { utilizado, sua dose } \\
\text { cumulativa, o uso prévio } \\
\text { de outros quimioterápicos } \\
\text { cardiotóxicos, e a } \\
\text { presença de outros fatores } \\
\text { de risco } \\
\text { cardiovasculares }{ }^{(3-7)} \text {. São } \\
\text { fatores de risco para } \\
\text { cardiotoxicidade de } \\
\text { quimioterápicos: } \\
\text { hipertensão, idade maior } \\
\text { que } 60 \text { anos, disfunção do } \\
\text { ventrículo esquerdo } \\
\text { prévia, irradiação torácica } \\
\text { prévia(7). }\end{array}$ \\
\hline $\begin{array}{l}\text { 5. Medicamentos de uso } \\
\text { diário }\end{array}$ & $\begin{array}{l}\text { Muitos indivíduos são } \\
\text { submetidos à polifarmácia } \\
\text { (número elevado de } \\
\text { medicamentos } \\
\text { diariamente), } \\
\text { objetivo de promover uma } \\
\text { melhor terapêutica } \\
\text { Entretanto, esta prática }\end{array}$ \\
\hline
\end{tabular}




\begin{tabular}{|l|l|}
\hline \hline & $\begin{array}{l}\text { manifestações clínicas da } \\
\text { síndrome }{ }^{(4-18)} .\end{array}$ \\
\hline manifestations & $\begin{array}{l}\text { Os sintomas } \\
\text { gastrointestinais são os } \\
\text { efeitos colaterais mais } \\
\text { comuns dos } \\
\text { quimioterápicos, } \\
\text { acontecendo em cerca de } \\
75 \% \text { dos pacientes que se } \\
\text { submetem a } \\
\text { quimioterapia. Desta } \\
\text { forma, onfermeiro que } \\
\text { atua neste cenário deve } \\
\text { estar atento e registrá-lo, } \\
\text { a fim de estratificar em } \\
\text { agudos, tardios e } \\
\text { antecipatórios e instituir a } \\
\text { terapêutica } \\
\text { medicamentosa } \\
\text { prescrita }{ }^{(3-18-10-26) .}\end{array}$ \\
\hline \hline
\end{tabular}

Fonte: dados da pesquisa.

\section{CONCLUSÃO}

Os casos de câncer no Brasil têm crescido consideravelmente e devido aos diversos tratamentos antineoplásicos, como a quimioterapia, têm aumentado a sobrevida dos pacientes. Porém a quimioterapia pode desenvolver reações tóxicas, como por exemplo, a cardiotoxicidade. Sendo assim, o conhecimento sobre os possíveis sinais e sintomas de toxidade cardíaca, bem como dos fatores de risco e adequado registro dessas informações pela enfermagem, tornam-se cada vez mais necessárias. Além disso, notou-se que são imprescindíveis para o cuidado adequado do paciente com câncer em tratamento quimioterápico, a identificação precoce do risco cardiovascular, o diagnóstico correto de alteração 1. Estimativa 2016: incidência de câncer no Brasil/Instituto Nacional de Câncer José de Alencar Gomes da Silva - Rio de Janeiro: INCA, 2015.
2. Leite MAC, Nogueira DA, Terra FS. Avaliação da autoestima em pacientes oncológicos submetidos a tratamento quimioterápico. Rev. Latino-Am. Enfermagem. 2015; 23(6):1082-1089.

3. Bonassa EMA, Gato MIR. Terapêutica Oncológica para Enfermagem e Farmacêuticos. 4 ed. São Paulo: Atheneu; 2012.

4. Adão, R, Keulenaerb G, Moreira AL, Silva CB. Cardiotoxicidade associado à terapêutica oncológica: mecanismos fisiopatológicos e estratégias de prevenção. Revista Portuguesa de Cardiologia. 2013; 32(5): 395-409.

5. Moretti MA, Herom RSR. Manual de cardiooncologia: manejo das terapias em oncologia e controle da cardiotoxidade. 1 ed. Rio de Janeiro: DOC content, 2016.

6. Martins WA, Moço ETM. Cardio-oncologia: o preço do envelhecimento. Revista Brasileira de Cardiologia. 2012; 25(3): 164-166.

7. Ramires JAF, Filho RK. Cardio-oncologia: temas comuns entre a cardiologia e a oncologia. São Paulo: editora Atheneu; 2014.

8. Araujo MM, Diniz SOS, Silva OS. Registros de enfermagem: reflexões sobre o cotidiano do cuidar. ABCS Health Sci. 2017; 42(3):161-165.

9. Ministério da Saúde (BR). Portaria no 529, de 1 de abril de 2013. Institui o Programa Nacional de Segurança do Paciente (PNSP). Diário Oficial [da] República Federativa do Brasil. 2013 abril 1; Seção 1. p. 19523- 49.

10. Guimarães RCR, Gonçalves RPF, Lima CA, Torres $M R$, Silva CSO. Ações de enfermagem frente às reações de quimioterápicos em pacientes oncológicos. Revista de Pesquisa Cuidado é Fundamental online [Internet].2015;7(2):24402452.

11. Miranda PC, Ferraz RRN, Barnabé AS, Fonseca SUL, Evengelista AA, AL Ramos, et al. A importância 


\section{ARTIGO ORIGINAL}

do registro de enfermagem em busca da qualidade. Gestão em foco. 2016.

12. Barral LNM, Ramos LH, Vieira MA, Dias OV, Souza SLP. Análise dos registros de enfermagem em prontuários de pacientes em um hospital de ensino. Rev. Min. Enferm. 2012; 16(2): 188-193.

13. Françolin L, Brito MFP, Gabriel CS, Monteiro TM, Bernardes A. A qualidade dos registros de enfermagem em prontuários de pacientes hospitalizados. Rev. enferm. UERJ. 2012; 20(1):7983.

14. Conselho Regional de Enfermagem do Rio Grande do Sul (BR). Lei no 590512 de julho de 1973. Normatiza os Registros de Enfermagem no Estado do Rio Grande do Sul. RS; 2016.

15. Conselho Nacional de Saúde (BR). Resolução 466, de 12 de dezembro de 2012. Diário Oficial da União. Ministério da Saúde. Brasília. DF; 13 de jun. 2013. Brasília. Seção 1: 59-61.

16. Karkow MC, Girardon-Perlini NMO, Mistura C, Rosa BVC, Rosa N, MS Martins, et al. Perfil dos usuários do serviço de radioterapia de um hospital universitário. Rev Enferm UFSM. 2013; 3(Esp.): 636646.

17. Sociedade Brasileira de Hipertensão. Exercício físico na redução da pressão arterial: Por quê? Como? Quanto? Revista hipertensão. 2017; 20(1): 3-15.

18. Silva FCM, Comarella L. Efeitos adversos associados à quimioterapia antineoplásica: levantamento realizado com pacientes de um hospital do estado do Paraná. Rev. UNIANDRADE. 2013; 14(3): 263-277.

19. Cruz FS, Rossato LG. Cuidados com o Paciente Oncológico em Tratamento Quimioterápico: o Conhecimento dos Enfermeiros da Estratégia Saúde da Família. Revista Brasileira de Cancerologia. 2015; 61(4): 335-341.
20. Oliveira $D$, Toledo $\sqcup$. Auditoria em enfermagem e qualidade da assistência à saúde: uma revisão de literatura [monografia]. Bragança Paulista: Universidade São Francisco; 2015.

21. Costa JM, Ramos RS, Santos MM, Silva DF, Gomes TF, Batista RQ. Complicações do estoma intestinal em pacientes em pós-operatório de ressecção de tumores de reto. Revista enfermagem atual 2017; Edição Especial: 35-42.

22. Fuziger HC. Registro de enfermagem: análise de prontuários de uma estratégia saúde da família[monografia]. Lajeado: Centro Universitário Univates; 2012.

23. Hoffmeister LV, Moura GMSS. Uso de pulseiras de identificação em pacientes internados em um hospital universitário. Rev. Latino-Am. Enfermagem. 2015; 23(1): 36-43.

24. Potter PA, Perry AG, Elkin MK. Procedimentos e intervenções de enfermagem. 2 ed. Rio de Janeiro: Elsevie, 2013.

25. Murassaki ACY, Versa GLGS, Bellucci JJA, Meireles VC, Vituri DW, Matsuda LM. Avaliação de cuidados na terapia intravenosa: desafio para a qualidade na enfermagem. Esc. Anna Nery [Internet]. 2013; 17(1): 11-16.

26. Lopes AL, Chammas R, lyeyasu H. Oncologia para a Graduação. 3 ed. São Paulo: Lemar, 2013. 